\title{
Comparing Encounter Characteristics Among Advanced Practice Clinicians and Physicians for Adult Same-Day Visits in Primary and Urgent Care
}

$\mathrm{J}$ Gen Intern Med 37(3):689-91

DOI: $10.1007 / \mathrm{s} 11606-021-06669-\mathrm{w}$

() Society of General Internal Medicine 2021

\section{BACKGROUND}

As the population ages amid growing shortages in primary care practitioners, expanding the supply of advanced practice clinicians (APC)—nurse practitioners and physician assistants - may offer a low-cost way to increase primary care access. ${ }^{1}$ Given differences in training, states have traditionally limited APC scope of practice, with 22 states requiring full physician oversight. Amid the coronavirus-19 pandemic, however, states have temporarily lifted these restrictions. ${ }^{2}$

There is debate regarding the care APCs provide. While APC visits may be associated with more diagnostic imaging than physician encounters, ${ }^{3}$ studies have shown comparable outcomes in regard to quality, patient experience, low-value care, and utilization. ${ }^{4}$ However, this work has focused on diagnoses amenable to algorithm-driven care. ${ }^{5}$

Little is known about the content of care physicians and APCs provide, specifically whether physicians handle more complex cases or provide additional services of value. Given changes under consideration to expand APC scope of practice and supply, it is critical to understand how this might impact care.

\section{OBJECTIVE}

This study aims to compare the practice of APCs to primary care physicians.

\section{METHODS AND FINDINGS}

We performed a retrospective cross-sectional analysis of adult same-day visits to primary and urgent care from December 2014 to August 2015 within our integrated health system. A total of 1200 unique patient encounters were randomly selected for chart review from 52 academic and community practice sites in urban, suburban, and rural settings. Two physicians

Received October 26, 2020

Accepted February 14, 2021

Published online March 3, 2021 classified reason for visit (acute complaint, stable chronic condition follow-up, exacerbation of chronic condition, preventive care, or other), encounter complexity (simple algorithmic v. complex diagnostic), complaint acuity (acute v. chronic), number of additional problems addressed, medication changes, and diagnostic interventions, including consultations, imaging, and labs (Table 1). Patient and clinician demographics were extracted from the electronic medical record. The Cleveland Clinic Institutional Review Board approved this study.

For patient and encounter measures, we reported frequencies (percentages) and measures of central tendency by provider type. We also described differences between physicians and APCs, using chi-square and $t$ tests. We modeled odds of prescription receipt, medication de-prescribing, and addressing additional concerns using mixed-effects logistic regression models, accounting for clustering by clinician. Models included provider, patient, and encounter characteristics.

Our sample included 393 physician and 807 APC visits (Table 2). Patients seeing APCs were more likely to be female $(68.9 \%$ v. $60.0 \%, p=0.002)$ and non-Caucasian $(19.6 \%$ v. $9.4 \%, p<.0001)$. APC visits were more often for simple algorithmic complaints than physician visits $(67.7 \%$ v $55.0 \%$, $p<.0001)$. Physicians more frequently addressed additional problems $(0.62$ v. $0.38, p=0.0003)$. The number of new medications prescribed was similar (1.26 v. $1.24, p=0.68)$, but physicians de-prescribed more medications $(0.48$ v. 0.34 , $p=0.02$ ). There was no difference in the number of diagnostic interventions $(2.89$ v. $2.84, p=0.84)$, including specialty consultations ( 1.24 v. $1.21, p=0.74)$. There was no difference in seeing an APC v. physician by setting (data not shown).

In the adjusted models, addressing additional complaints (aOR 1.80, 95\% CI 1.27-2.57) and de-prescribing (aOR 1.44, 95\% CI 1.07-1.93) were associated with seeing a physician v. an APC.

\section{DISCUSSION}

In this observational review of 1200 cases, we found small differences in practice between APCs and physicians. APCs more often saw simple, algorithmic complaints, while physicians were more likely to address additional health concerns or de-prescribe medications. Both prescribed medications and ordered diagnostic interventions at similar rates. To our 
Table 1 Classification Scheme for Acute Patient Complaints by Complexity

\begin{tabular}{ll}
\hline \hline Minor illnesses amenable to algorithm-guided care* & Illnesses requiring diagnostic acumen \\
\hline 1. Skin injury & 1. Abdominal pain \\
2. Upper respiratory symptoms & 2. Chest pain \\
3. Sore throat & 3. Dizziness \\
4. Lower urinary symptoms & 4. Syncope \\
5. Acute diarrhea & 5. Glycemic control \\
6. Low back pain & 6. Joint pain \\
7. Increased arterial pressure (blood pressure) & 7. Shortness of breath \\
8. Pink eye (conjunctivitis) & 8. Palpitations \\
9. Burns & 9. Edema \\
10. Tooth pain & 10. Ulcer \\
11. Twisted ankle & 11. Urinary incontinence \\
12. Emergency contraception & 12. Headache/migraine \\
13. Anxiety attacks & 13. Heartburn \\
14. Skinfold dermatitis & 14. Arthritis \\
15. Influenza & 15. Fatigue \\
16. Nosebleeds & 16. Leg pain \\
& 17. Weight gain \\
& 18. Weight loss \\
& 19. Depression \\
\hline
\end{tabular}

*Classification scheme adopted from a program of nurse algorithm-guided care for adult patients ${ }^{5}$

Table 2 Patient and Encounter Characteristics for All Same-Day Visits, 2014-2015

\begin{tabular}{|c|c|c|c|}
\hline Variables & Physicians ( $n=393$ visits) & Advanced practice clinicians ( $n=807$ visits) & $p$ value \\
\hline \multicolumn{4}{|l|}{ Patient characteristics } \\
\hline Male, $n(\%)$ & $157(40.0)$ & $251(31.1)$ & 0.002 \\
\hline Race, $n(\%)$ & & & $<.0001$ \\
\hline Caucasian & $356(90.6)$ & $649(80.4)$ & \\
\hline African American & $15(3.2)$ & $113(14.0)$ & \\
\hline Other & $22(5.7)$ & $45(5.6)$ & \\
\hline Age, y (SD) & $48.7(17.7)$ & $49.1(17.3)$ & 0.72 \\
\hline \multicolumn{3}{|l|}{ Insurance, $n(\%)$} & 0.0001 \\
\hline Commercial & $244(60.1)$ & $559(69.3)$ & \\
\hline Medicare & $74(18.8)$ & $141(17.5)$ & \\
\hline Medicaid & $58(14.8)$ & $57(7.1)$ & \\
\hline Other* & $17(4.3)$ & $50(6.2)$ & \\
\hline Marital status, $n(\%)$ & $229(58.3)$ & $435(53.9)$ & 0.15 \\
\hline Number of medications taking prior to visit, $n$ (SD) & $5.4(4.9)$ & $6.1(5.3)$ & 0.02 \\
\hline Current prescription medications ${ }^{\dagger}$ & $3.8(3.3)$ & $3.9(3.4)$ & 0.63 \\
\hline Current over-the-counter medications ${ }^{\dagger}$ & $1.4(1.5)$ & $1.6(1.6)$ & 0.06 \\
\hline Current supplements ${ }^{\dagger}$ & $1.2(1.6)$ & $1.4(1.8)$ & 0.04 \\
\hline \multicolumn{4}{|l|}{ Encounter characteristics } \\
\hline Setting, $n(\%)$ & & & 0.76 \\
\hline Primary care & $199(50.6)$ & $401(49.7)$ & \\
\hline Urgent care & $194(49.4)$ & $406(50.3)$ & \\
\hline \multicolumn{3}{|l|}{ Reason for visit, $n(\%)$} & 0.40 \\
\hline Acute condition/symptom & $330(84.0)$ & $703(87.1)$ & \\
\hline Preventive care & $1(0.3)$ & $2(0.3)$ & \\
\hline Chronic condition/follow-up & $46(11.7)$ & $83(10.3)$ & \\
\hline Exacerbation & $13(3.3)$ & $17(2.1)$ & \\
\hline Other & $3(0.8)$ & $2(0.3)$ & \\
\hline \multicolumn{3}{|l|}{ Primary complaint type, $n(\%)$} & $<.0001$ \\
\hline Simple/algorithmic problem & $216(55.0)$ & $546(67.7)$ & \\
\hline Complex/diagnostic problem & $177(45.0)$ & $261(32.3)$ & \\
\hline \multicolumn{4}{|c|}{ Encounters with patients taking high-risk medications, $n(\%)$} \\
\hline Narcotics & $47(12.0)$ & $101(12.5)$ & 0.78 \\
\hline Warfarin & $14(3.6)$ & $17(2.1)$ & 0.14 \\
\hline Insulin & $14(3.6)$ & $30(3.7)$ & 0.89 \\
\hline Oral hypoglycemic (except metformin) & $15(3.8)$ & $35(4.3)$ & 0.67 \\
\hline Number of additional problems addressed, $n$ (SD) & $0.6(1.2)$ & $0.4(0.8)$ & 0.0003 \\
\hline Number of new medications prescribed, $n$ (SD) & $1.3(0.8)$ & $1.2(0.8)$ & 0.68 \\
\hline \multicolumn{4}{|c|}{ Encounters involving starting new high-risk medications, $n(\%)$} \\
\hline Narcotics & $11(2.8)$ & $18(2.2)$ & 0.55 \\
\hline Warfarin & $0(0)$ & $0(0)$ & $\mathrm{n} / \mathrm{a}$ \\
\hline Insulin & $0(0)$ & $1(0.1)$ & 0.59 \\
\hline
\end{tabular}


Table 2. (continued)

\begin{tabular}{|c|c|c|c|}
\hline Variables & Physicians ( $n=393$ visits) & Advanced practice clinicians ( $n=807$ visits) & $p$ value \\
\hline Oral hypoglycemic (except metformin) & $0(0)$ & $2(0.3)$ & 0.32 \\
\hline Number of medications stopped, $n$ (SD) & $0.5(1.0)$ & $0.3(0.9)$ & 0.02 \\
\hline Total number of interventions ordered, $n$ (SD) & $2.9(2.9)$ & $2.8(2.9)$ & 0.84 \\
\hline Number of consults placed & $1.2(0.5)$ & $1.2(0.5)$ & 0.74 \\
\hline
\end{tabular}

*Other includes TRICARE, self-pay, and worker's compensation

${ }^{\dagger}$ Mean number of current medication subtypes or supplements provided for those patients endorsing taking at least one medication prior to encounter Bold values are statistically significant at $p<0.05$

knowledge, our study is the first to examine differences in $\mathrm{APC}$ and physician practice content.

Prior studies finding similar quality of care between APCs and physicians did not assess the complexity of decisionmaking, ${ }^{6}$ focusing instead on diagnoses amenable to algorithmic care, such as low-back pain and sinusitis. [1-6] Although our system employs no formal triage process, physicians saw a higher proportion of visits requiring diagnostic acumen. Physicians also added value by de-prescribing and addressing additional complaints.

Limitations include the single institution sample, which may not be representative. We also did not assess diagnostic accuracy or longitudinal outcomes.

Given primary care shortages and rising care demand, it may be tempting to replace physicians with APCs. Our findings suggest that they often do similar work, but physicians may add value in unmeasured ways. Systems are needed to ensure that work is divided between physicians and APCs in ways that maximize efficiency through the use of each practitioner's particular skills.

Jessica A. Hohman, MD, MSc, MSc ${ }^{1,2}$

Aditi Patel, $M D^{3}$

Parth Parikh, $M D^{4}$

Michael B. Rothberg, $M D, M P H^{1,2}$

${ }^{1}$ Cleveland Clinic Center for Value-Based Care Research,

Cleveland, OH, USA

${ }^{2}$ Cleveland Clinic Community Care,

Cleveland, OH, USA

${ }^{3}$ Department of Rheumatic and Immunologic Diseases, Cleveland Clinic Orthopedic and Rheumatic Institute,

Cleveland, OH, USA

${ }^{4}$ Department of Cardiovascular Medicine, Cleveland Clinic Heart and Vascular Institute,

Cleveland, $\mathrm{OH}$, USA
Corresponding Author: Jessica A. Hohman, MD, MSc, MSc; Cleveland Clinic Community Care, Cleveland, $\mathrm{OH}$, USA

\section{Declarations:}

Conflict of Interest: The authors declare that they do not have a conflict of interest.

\section{REFERENCES}

1. Roblin DW, Howard DH, Becker ER, Kathleen Adams E, Roberts MH. Use of midlevel practitioners to achieve labor cost savings in the primary care practice of an MCO. Health services research. 2004;39(3):607-26.

2. Bluth R. California Shies Away From Calls to Eliminate Restrictions on Nurse Practitioners. COVID-19: Kaiser Health News; 2020.

3. Hughes DR, Jiang M, Duszak R. A comparison of diagnostic imaging ordering patterns between advanced practice clinicians and primary care physicians following office-based evaluation and management visits. JAMA internal medicine. 2015;175(1):101-7.

4. Voils CI, Venne VL, Weidenbacher H, Sperber N, Datta S. Comparison of telephone and televideo modes for delivery of genetic counseling: a randomized trial. Journal of genetic counseling. 2018;27(2):339-48.

5. Fabrellas N, Sánchez C, Juvé E, Aurin E, Monserrat D, Casanovas E, et al. A program of nurse algorithm-guided care for adult patients with acute minor illnesses in primary care. BMC family practice. 2013;14(1):61.

6. Mafi JN, Wee CC, Davis RB, Landon BE. Comparing use of low-value health care services among US advanced practice clinicians and physicians. Annals of internal medicine. 2016;165(4):237-44.

Publisher's Note: Springer Nature remains neutral with regard to jurisdictional claims in published maps and institutional affiliations. 